\title{
A biological route to conjugated alkenes: microbial production of hepta-1,3,5-triene
}

Hanan L. Messiha ${ }^{\dagger}$, Karl A. P. Payne ${ }^{\dagger \S}$, Nigel S. Scrutton ${ }^{\dagger \S}$ and David Leys ${ }^{\dagger}$

${ }^{\dagger}$ Manchester Institute of Biotechnology, Department of Chemistry, School of Natural Sciences, Faculty of Science and Engineering, The University of Manchester, 131 Princess Street, Manchester, M1 7DN, United Kingdom

${ }^{\S}$ Future Biomanufacturing Research Hub (Future BRH), Manchester Institute of Biotechnology, The University of Manchester, 131 Princess Street, M1 7DN, United Kingdom 


\section{Table of Contents}

Fig S1. Mechanism for priming and chain elongation in type II PKS 3

1. Additional Experimental Details $\quad 4$

1.1 Materials and Reagents $\quad 4$

Table S1. Primers used for generating del_AdmH-AdmP, Fdc1/UbiX, PKS-ABCDER-ptrc constructs 5

Table S2. Plasmids, cosmids and strains used in this study

1.2. Construction of the del_AdmH-AdmP construct $\quad 6$

Fig S2. Map of the del_AdmH-AdmP construct $\quad 6$

Fig S3. Agarose gel electrophoresis analysis of the del_AdmH-AdmP construct 6

Fig S4. Map of the cloned AnFdc1EcUbiXpBbA1C

1.3. Construction of the PKS-ABCDER-native promoter and PKS-ABCDER-pTre constructs 8

1.4. Expression of PKS-ABCDER-native promoter and PKS-ABCDER-pTre constructs in

Fig S5. Maps of the constructed PKS-ABCDER, PKS-ABCDER-native promoter,

PKS-ABCDER-native promoter in pBbA1K $\mathrm{c}$ and PKS-ABCDER-pTre in pBbE1K construct 9

2. Results

2.1 Sequence analysis of cosmid 2194C1

Fig S6. Cosmid 2194C1 map of the andrimid gene cluster of Pantoea agglomerans in SuperCos 1 vector

Fig S7. SDS-PAGE analysis illustrates the expression of cosmid 2194C1 in XL1-Blue MR cells

2.2 LC/MS analysis

Fig S8. Mass spectra of the products of cosmid 2194C1 (in Fig 3A)

\subsection{GC/MS data}

Fig S9. Mass spectra of hepta-1,3,5-triene product (in Fig 3B, Fig 3 C) analysed by GC/MS in the gaseous phase

Fig S10. Mass spectra of hepta-1,3,5-triene product analysed by GC/MS in the liquid phase

Fig S11. Standard curve of hepta-1,3,5-triene measured by GC/MS

\subsection{HPLC Analysis}

Fig S12. Standard curve of 2,4,6-octatrienoic acid measured by HPLC

Fig S13. HPLC analysis showing the Fdc1 catalysed decarboxylation of the 2,4,6-octatrieonic acid product of cosmid $2194 \mathrm{C} 1$ in vivo

Fig S14. HPLC analysis of the products from the expression of the del AdmH-AdmP construct in XL1-Blue MR in the absence and presence of cerulenin

Fig S15. HPLC analysis of the products from the expression of the PKS-ABCDER-native promoter construct in pBbA1K-RFP vector and the co-expression with Bacillus subtilis Sfp in BL21(DE3) cell 

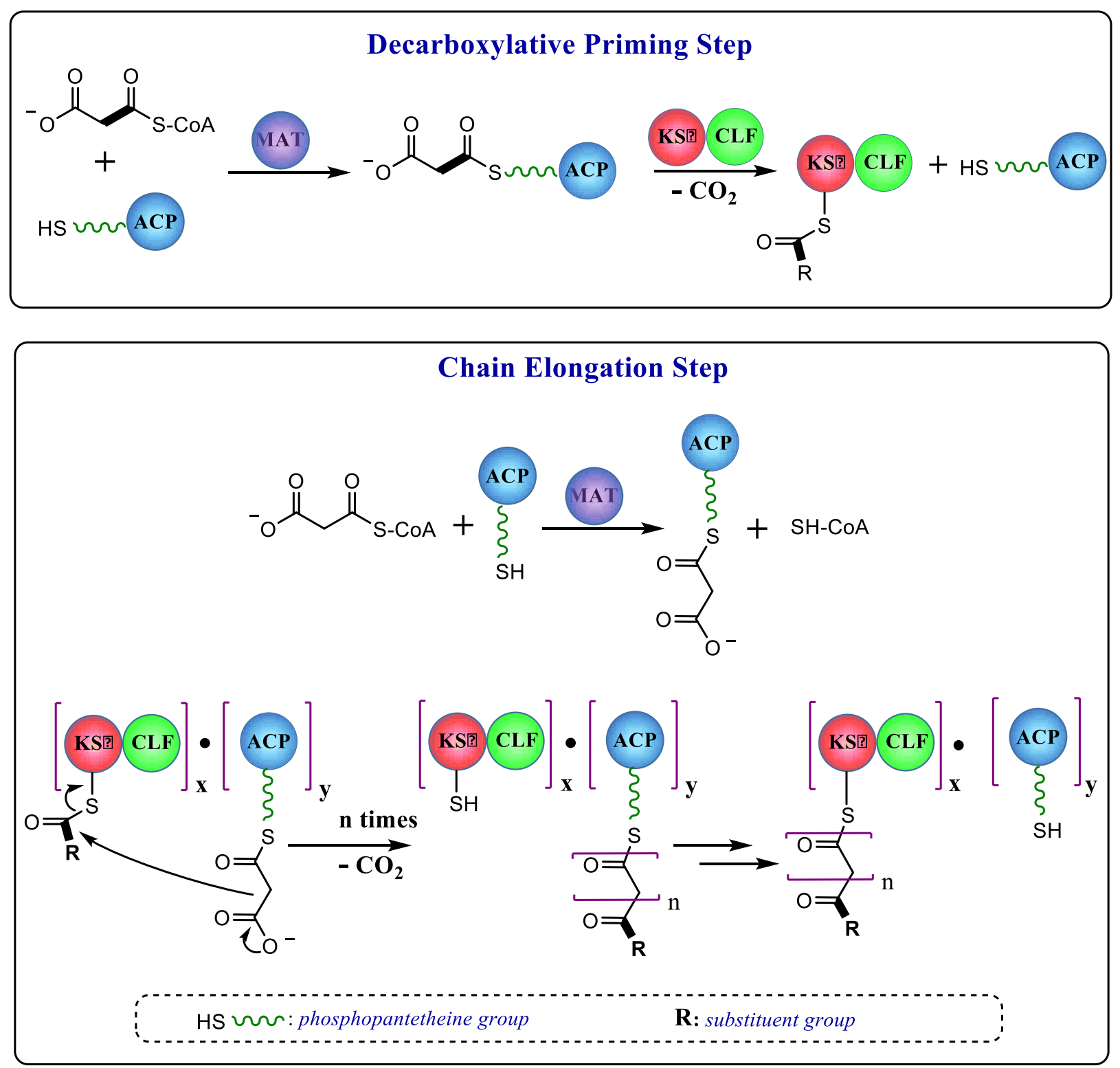

Figure S1. Mechanism for priming and chain elongation in type II PKS. ACP, acyl carrier protein; KS, ketosynthase (KS $\alpha$ ), CLF, chain-length factor (also $\mathrm{KS}_{\beta}$ ), MAT, malonyl-CoA:ACP acyltransferase (adapted after modification from Hertwek et al, 2007 \& Zhang and Tang, 2009) $)^{1,2}$. 


\section{Additional Experimental Details}

\subsection{Materials and reagents}

All chemicals, reagents, organic solvents and other materials used in this study were of analytical grade and obtained from Sigma-Aldrich Co Limited, Fisher Scientific UK Limited, Agilent Technologies UK Limited, Roche Applied Sciences, and VWR International Limited. Media components were obtained from Formedium (Norflok, UK). The 2,4,6-ctatrienoic acid standard was synthesized by Sigma-Aldrich Co Limited. Reagents and solvents used for HPLC, GC/MS and LC/MS, analysis are of HPLC grade. The Escherichia coli (E. coli) strains used in this study were obtained from various suppliers: XL1-Blue MR supercompetent cells from Stratagene. The One Shot ${ }^{\mathrm{TM}}$ TOP10 chemically competent cells and the NEB ${ }^{\circledR}$ 5-alpha competent cells were from New England BioLabs Inc. The pBbA1C vector from the BgIBrick series of vectors ${ }^{3}$ was obtained from Addgene. CloneAmp HiFiPCR premix (Clontech) and the In-Fusion HD Cloning Plus kit was obtained from Takara Bio Inc., The Q5® High-Fidelity DNA Polymerase and DpnI from New England BioLbs Inc. and the QIAquick PCR purification kit from Qiagen. Gene sequencing and oligonucleotides syntheses were performed by Eurofins MWG (Ebersberg, Germany). Cosmid 2194C1 in SuperCos 1 vector was obtained from Dr Fumihiro Ishikawa, Faculty of Pharmacy, Kindai University, Japan. Sequence analysis of cosmid 2194C1 in SuperCos 1 vector was performed by genome sequencing of TOP 10 E. coli cells harboring cosmid 2194C1 in SuperCos 1 vector at Microbes NG, University of Birmingham. LC/MS analysis was performed at the LC/MS facility at the Manchester Institute of Biotechnoloy (MIB).

All primers used in this study are listed in Table S1, plasmids, cosmids, vectors and strains are in Table S2. 


\begin{tabular}{|l|l|}
\hline Primers & Sequence \\
\hline PKS_dHP-F & atactcagtttaaaatcaatctgagtttgacagtagaatgtgacaag \\
\hline PKS_dHP-R & ttttaaactgagtatttccaaactaattggcacattcc \\
\hline C PCR-F1 & cgattcgtgtggaggacactacaggatgttc \\
\hline C PCR-F2 & cattgccttacacgctgagcggtaaagtcgac \\
\hline C PCR-R & cattaatgccagcagagacacatcaggcggtac \\
\hline AnFdcF & ggagatatacatatgatgtcggctcaaccggcc \\
\hline AnFdcXR & actcgagtttggatccctaattcgagaagcccattttgg \\
\hline AnFdc1EcXF & ctcgaattagggatccacaaggaggaatcgacatgaaacgactcattgtaggc \\
\hline EcXR & gagatccttactcgagttatgcgccctgccagcg \\
\hline
\end{tabular}

Table S1. Primers used for generating del_AdmH-AdmP, Fdc1/UbiX , PKS-ABCDER-ptrc constructs.

\begin{tabular}{|c|c|}
\hline Cosmid, plasmids, vectors & Source \\
\hline $\begin{array}{l}\text { Cosmid 2194C1 } \\
\text { (GenBank: accession number AY192157) } \\
\text { (Fig S6) }\end{array}$ & $\begin{array}{l}\text { obtained from Dr Fumihiro Ishikawa, } \\
\text { Japan }\end{array}$ \\
\hline del_AdmH-AdmP (Fig S3) & This study \\
\hline AnFdc1pET30a & Ref 5 \\
\hline EcUbiXpET21b & Ref 5 \\
\hline pBbA1c-RFP & Addgene, Ref 4 \\
\hline AnFdc1pBbA1C & This study \\
\hline EcUbiXpET21b & This study \\
\hline AnFdc1EcUbiXpBbA1c (Fig S5) & This study \\
\hline PKS-ABCDER-native promoter & This study \\
\hline PKS-ABCDER-pTrc & This study \\
\hline pCDF1b-Sfp & Ref 5 \\
\hline pBbA1K-RFP & Addgene, Ref 4 \\
\hline pBbE1K-RFP & Addgene, Ref 4 \\
\hline \multicolumn{2}{|l|}{ Strains } \\
\hline One Shot ${ }^{\mathrm{TM}} \mathrm{TOP} 10$ & Stratagene \\
\hline NEB® 5-alpha & New England BioLabs Inc. \\
\hline XL1-Blue MR supercompetent & Stratagene \\
\hline $\mathrm{BL21(DE3)}$ & Novagen \\
\hline JM109 & Promega \\
\hline
\end{tabular}

Table S2. Cosmid, plasmids and strains used in this study. 


\subsection{Construction of the del_AdmH-AdmP construct}

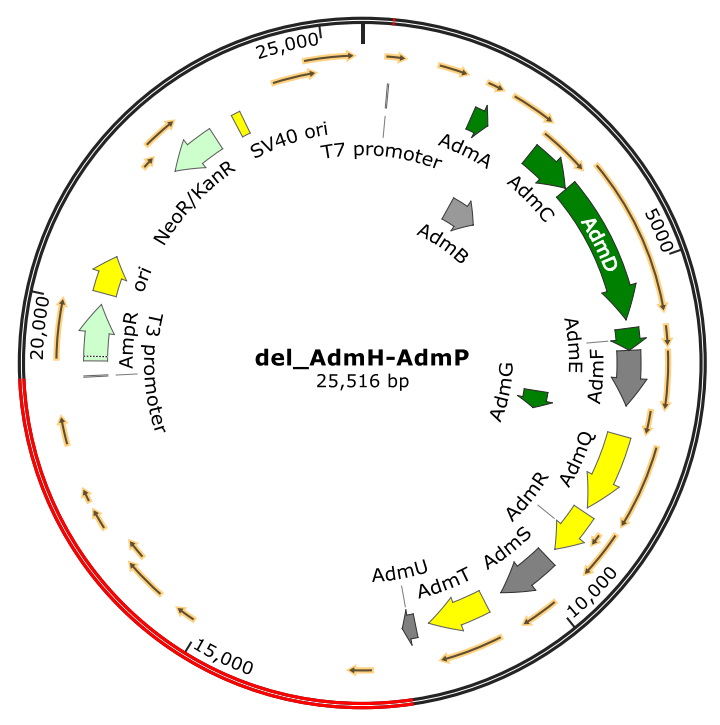

Figure S2. Map of the del_AdmH-AdmP construct

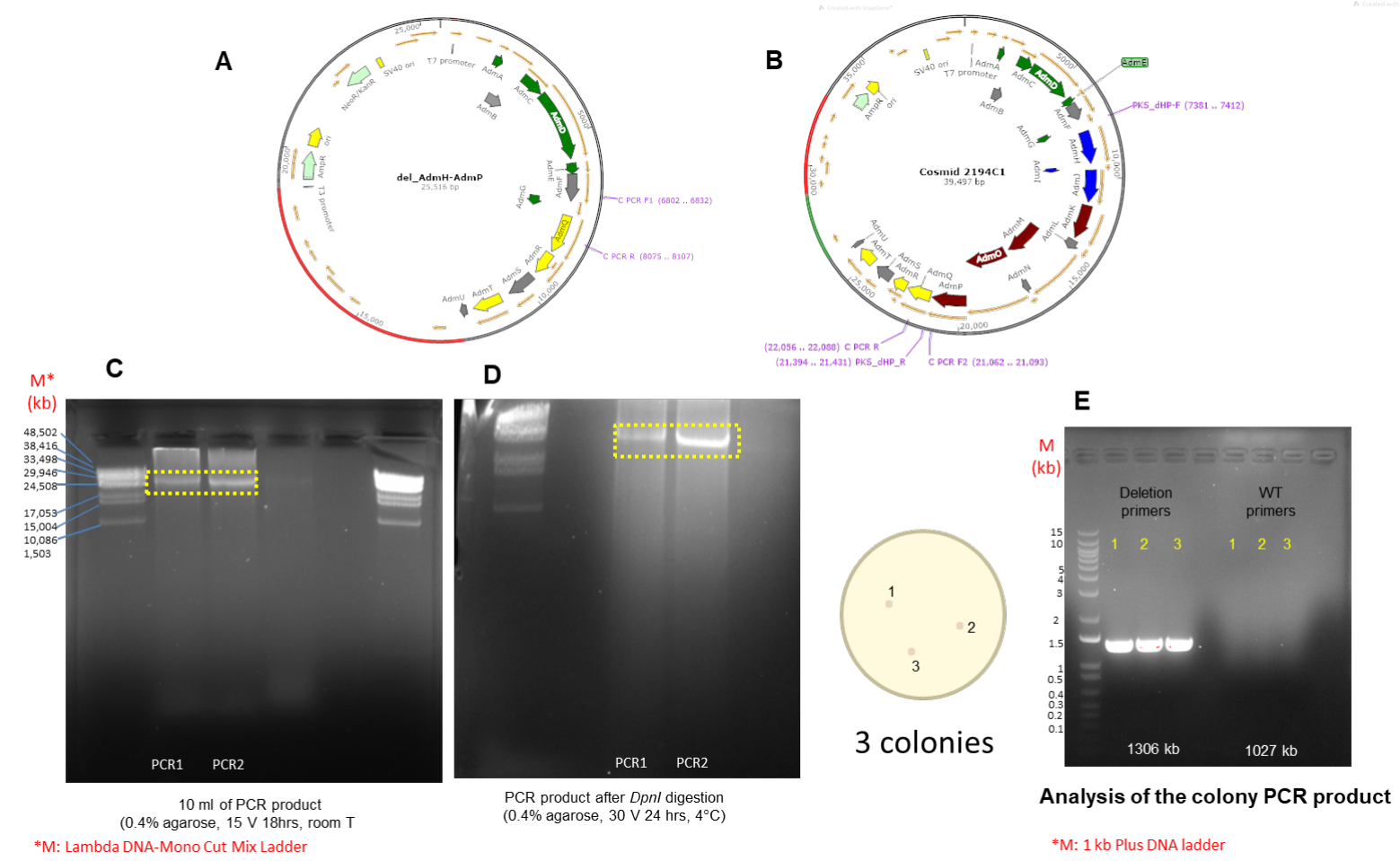

Figure S3. Agarose gel electrophoresis analysis of the del_AdmH-AdmP construct. The PCR product (PCR1, PCR2 as shown in C, D are duplicate reactions). These were obtained from deleting the seven genes of cosmid 2194C1 (B) as described in the "Methods" section to construct del_AdmH-AdmP (A). Primers were added to illustrate the fragment amplified. E shows the colonies PCR product analysis confirming the deletion of the seven genes in the three colonies obtained. 


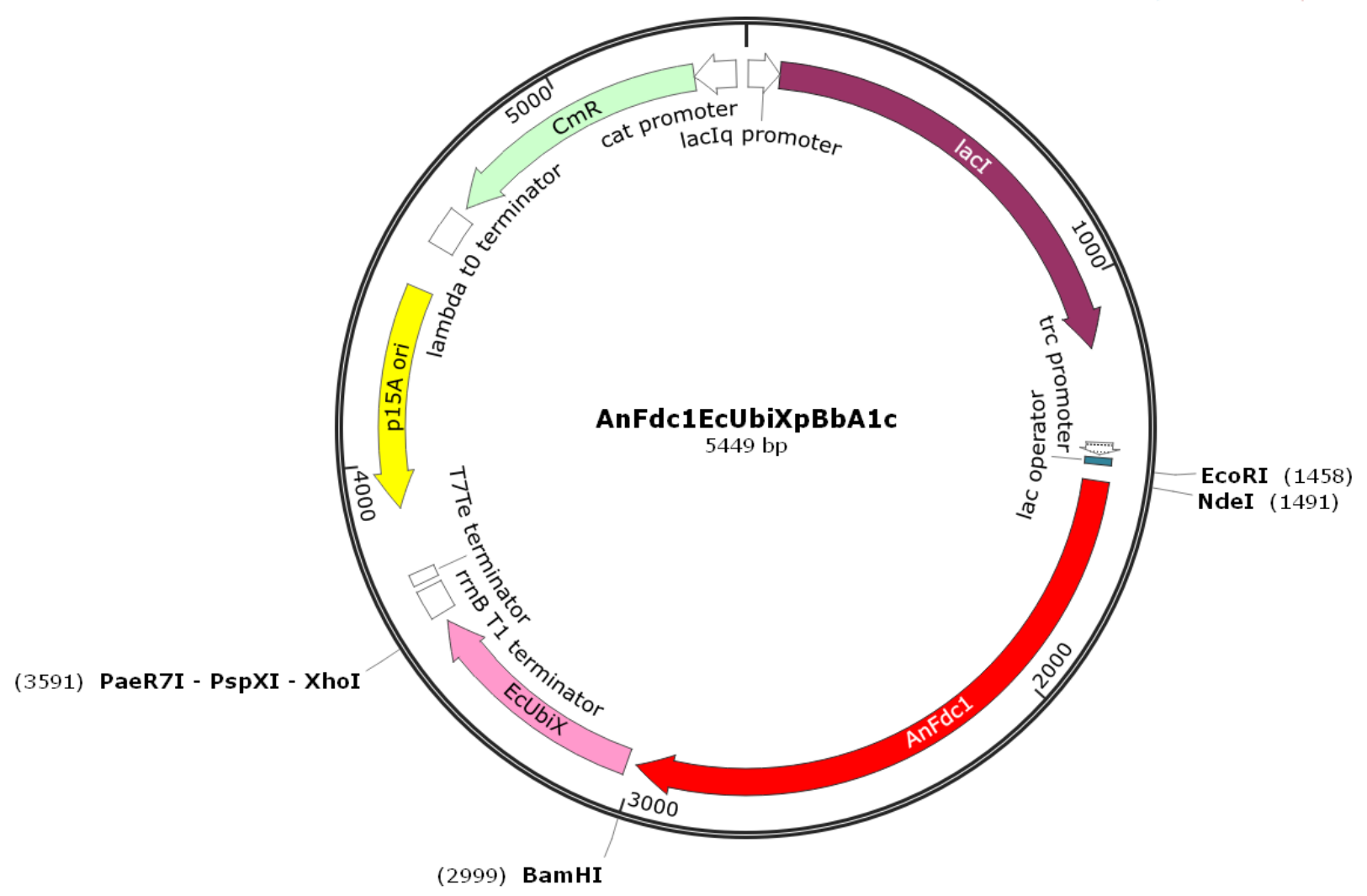

Figure S4. Map of the cloned AnFdc1EcUbiXpBbA1c. See "Methods" section for details. 


\subsection{Construction of the PKS-ABCDER-native promoter and PKS-ABCDER-pTrc constructs}

The "PKS-ABCDER-native promoter" construct was synthesised and subcloned into the pBbA1K-RFP vector at Invitrogen GeneArt, ThermoFisher Scientific. PKS-ABCDER (Fig S5) is a short cassette that includes the predicted genes essential to produce 2,4,6-octatrienoic acid. These are the acyl carrier protein AdmA, the ketosynthase/chain length factor AdmD, the ketoreductase AdmC, the dehydratase AdmE, and the phosphopantetheinyl transferase AdmR with including $200 \mathrm{bp}$ upstream the AdmR gene. In addition, AdmB was included in the cassette as its gene sequence overlaps with AdmC sequence. The "PKS-ABCDER-native promoter" construct in pBbA1K-RFP vector includes in addition to the PKS-ABCDER the $1314 \mathrm{bp}$ native sequence upstream the AdmA of cosmid 2194C1 (Fig S5). In this construct the expression of the genes is derived by the native constitutive promoter of cosmid $2194 \mathrm{C} 1$.

The "PKS-ABCDER-pTrc" construct was also synthesised and subcloned into pBbE1K-RFP vector at Invitrogen GeneArt, ThermoFisher Scientific (Fig S5). This construct includes the PKS-ABCDER construct and the expression of the genes is derived by the pTrc promoter of the vector.

Both constructs did not result in any production of 2,4,6-octatrienoic acid when expressed in various E. coli strains (B121, JM109, XL1-Blue MR) and the SDS PAGE analysis did not show any signs of expression compared to the control cells; even when the expression was attempted under the regulation of the inducible pTrc promoter.

We have also tested the co-expression of additional phosphopantetheinyl transferase (Bacillus subtilis Sfp) in case AdmR might not have been expressed. We therefore co-expressed PKSABCDER-native promoter construct in pBbA1K-RFP vector with the Bacillus subtilis Sfp (pCDF1b-Sfp) construct ${ }^{4}$. The later was previously constructed in our laboratory for the expression of Sfp;phosphpantetheine transferase from Bacillus subtilis.

\subsection{Expression of PKS-ABCDER-native promoter and PKS-ABCDER-pTre constructs in E. coli cells and testing the production of 2,4,6-octatrienoic acid}

The PKS-ABCDER-native promoter construct was transformed into various E. coli strains (XL1-Blue MR, BL21(DE3), JM109 cells). A single colony was used to inoculate $5 \mathrm{~mL}$ of LB medium containing ampicillin $(100 \mu \mathrm{g} / \mathrm{ml})$ and kanamycin $(30 \mu \mathrm{g} / \mathrm{ml})$ and grown overnight, shaking at $37^{\circ} \mathrm{C}$. The inoculum was transferred and grown in $2 \mathrm{~L}$ flasks, containing LB medium $(0.5 \mathrm{~L})$ with ampicillin $(100 \mu \mathrm{g} / \mathrm{ml})$ and kanamycin $(30 \mu \mathrm{g} / \mathrm{ml})$ at $30^{\circ} \mathrm{C}$ for 18 hours in a shaking incubator at $180 \mathrm{rpm}$. The PKS-ABCDER-pTrc construct was similarly transformed into E. coli BL21(DE3) cells and cultures were grown in LB media $(0.5 \mathrm{~L})$ containing ampicillin (100 $\mu \mathrm{g} / \mathrm{ml})$ and kanamycin $(30 \mu \mathrm{g} / \mathrm{ml})$ at $30^{\circ} \mathrm{C}$ until an $\mathrm{OD}_{600 \mathrm{~nm}}$ of 0.6 . IPTG was added at $0.5 \mathrm{mM}$ and the culture were grown for 18 hours in a shaking incubator at $180 \mathrm{rpm}$. For the coexpression of the PKS-ABCDER and Sfp constructs, the PKS-ABCDER-native promoter construct and the pCDF1b-Sfp were co-expressed in BL21(DE3) cells. Cells were grown in LB media $(0.5 \mathrm{~L})$ containing ampicillin $(100 \mu \mathrm{g} / \mathrm{ml})$, kanamycin $(30 \mu \mathrm{g} / \mathrm{ml})$ and spectinomycin $(50$ $\mu \mathrm{g} / \mathrm{ml})$ at $30^{\circ} \mathrm{C}$ until on $\mathrm{OD}_{600 \mathrm{~nm}}$ of 0.6 . IPTG was added at $0.25 \mathrm{mM}$ concentration and the cultures were grown overnight in a shaking incubator at $180 \mathrm{rpm}$ at $30^{\circ} \mathrm{C}$. 
In all, $150 \mathrm{ml}$ of cell cultures were extracted in $100 \mathrm{ml}$ ethyl acetate twice. The ethyl acetate extract was evaporated to dryness and the residue was dissolved in $2 \mathrm{ml}$ of $50 \%$ methanol/water (HPLC grade). The products were analysed by HPLC as described in the "Methods" section to test the production of 2,4,6-octatrienoic acid. An example of the results is shown at the HPLC analysis section, section 2.4, Fig S15.

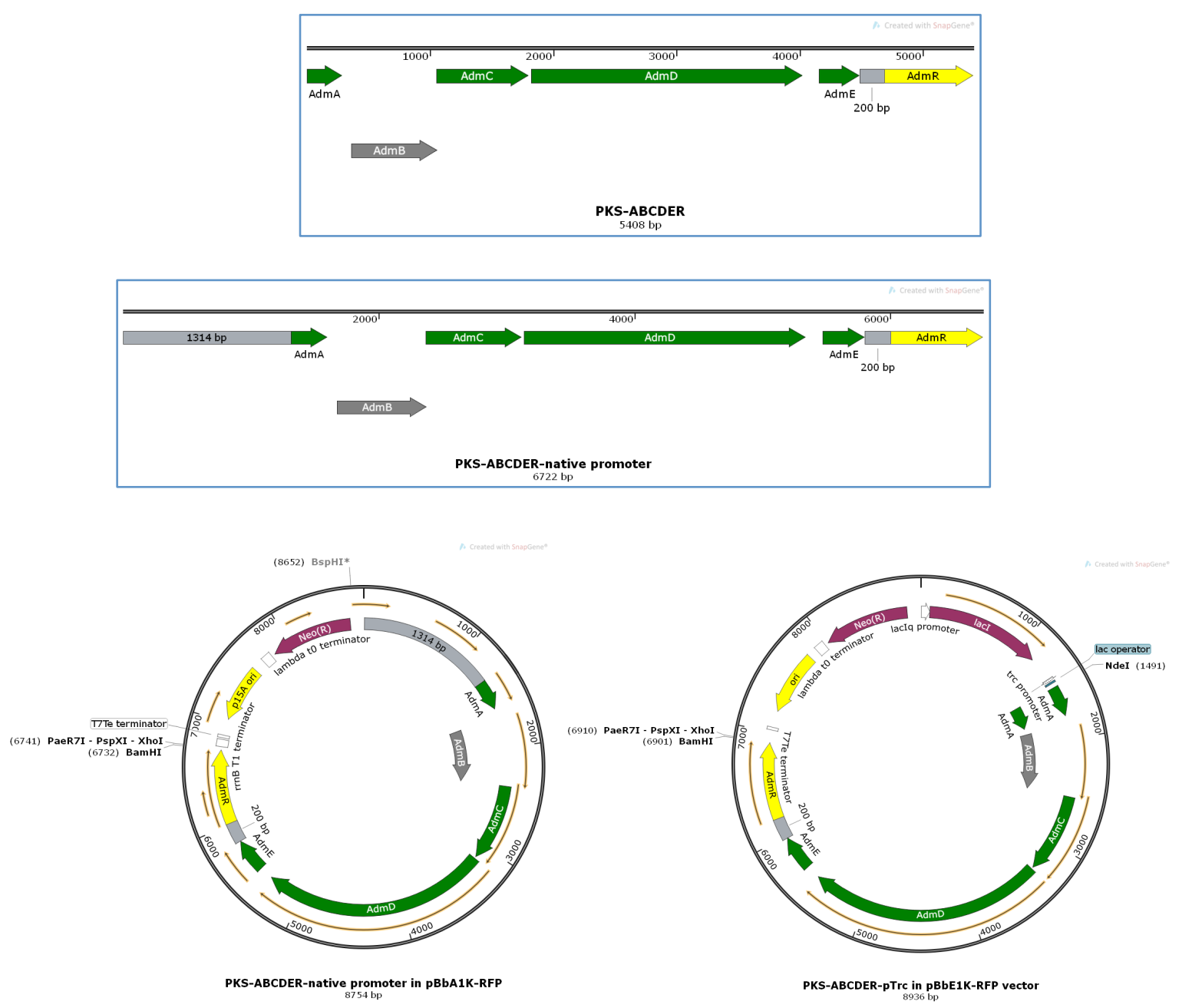

Figure S5. Maps of the constructed PKS-ABCDER, PKS-ABCDER-native promoter, PKSABCDER-native promoter in pBbA1K $\mathrm{c}$ and PKS-ABCDER-pTrc in pBbE1K constructs. 


\section{Results}

\subsection{Sequence analysis of cosmid 2194C1}

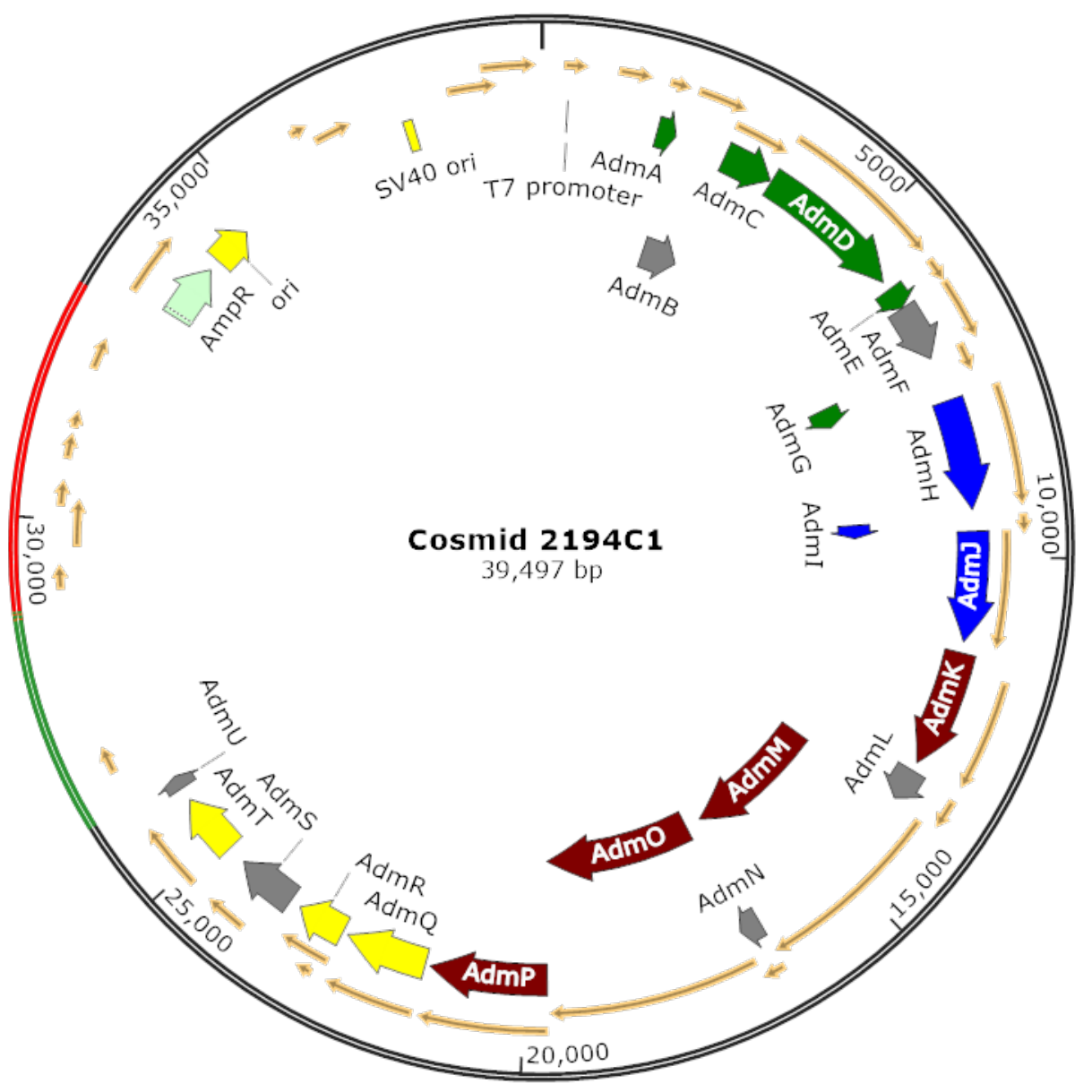

Figure S6. Cosmid 2194C1 map of the andrimid gene cluster of Pantoea agglomerans in SuperCos 1 vector. Sequence analysis confirmed the sequence of the 21 genes involved in the andrimid biosynthesis. The sequence analysis was carried out at the genome sequencing service, MicrobesNG, University of Birmingham, UK. Cosmid 2194C1 (GenBank: accession number AY192157). ${ }^{5}$ 


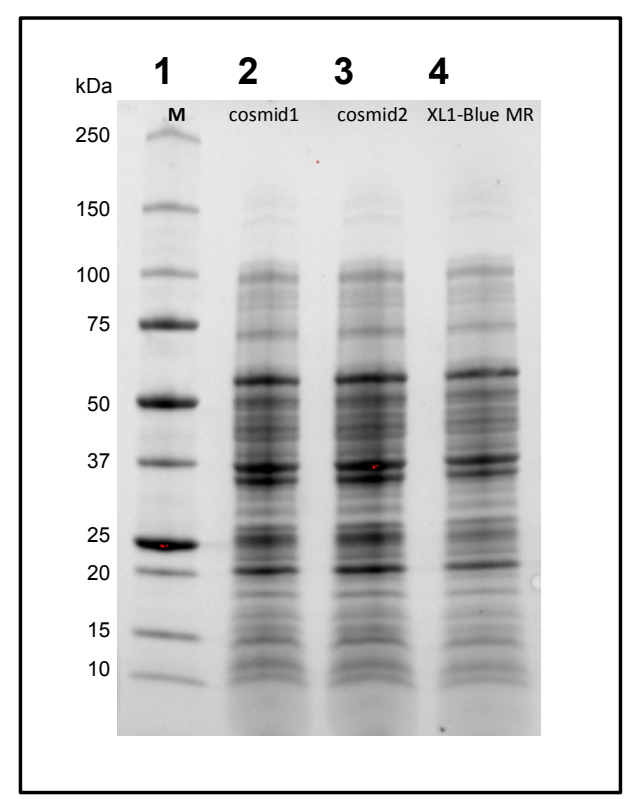

Figure S7. SDS-PAGE analysis illustrates the expression of cosmid 2194C1 in XL1-Blue MR cells. Samples were run on 4-20\% Mini-PROTEAN TGX Stain-Free gel from BioRad. Protein Marker: Precision Plus Protein unstained. Lanes 2,3 pellets of cosmid 21941C expressed in XL1-Blue MR cells as described in the "Methods" section. Lane 4 is pellet sample from control XL1-Blue MR cells. 


\subsection{LC/MS analysis}

Figure S8. Mass spectra of the products of cosmid 2194C1 (in Fig 3A). Three products were identified. Product 1 is trans-cinnamic acid, 2 is 2,4,6-octatrienoic acid and 4 is andrimid. Product 3 was difficult to identify (MS data is shown below).

\section{trans-cinnamic acid MS data (product 1 in Fig 3A)}

\section{(negative ion mode)}

The calculated monoisotopic mass of cinnamic acid is $148.05242\left(\mathrm{C}_{9} \mathrm{H}_{8} \mathrm{O}_{2}\right)$

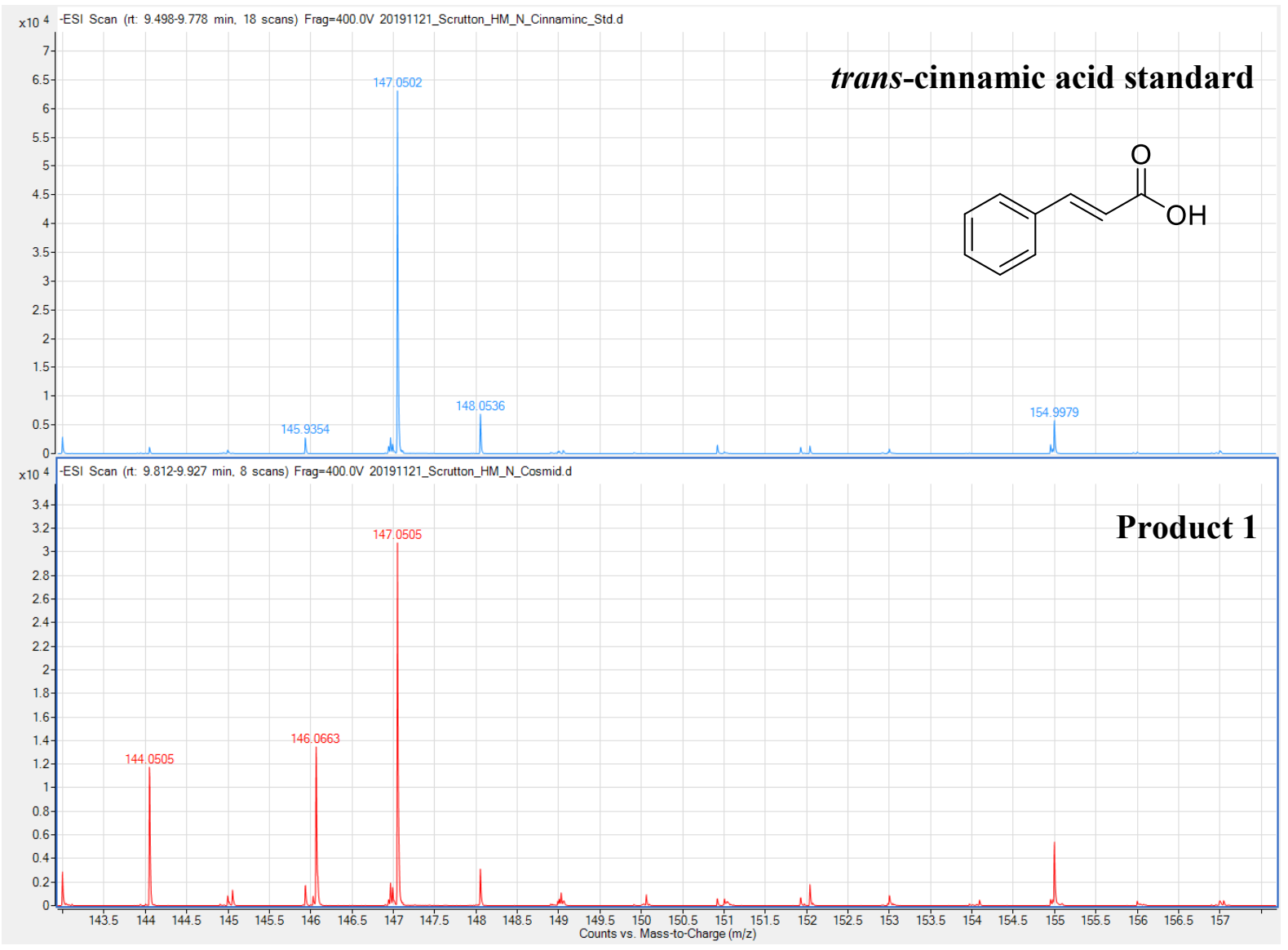


trans-cinnamic acid MS data (product 1 in Fig 3A)

(positive ion mode)

The calculated monoisotopic mass of cinnamic acid is $148.05242\left(\mathrm{C}_{9} \mathrm{H}_{8} \mathrm{O}_{2}\right)$

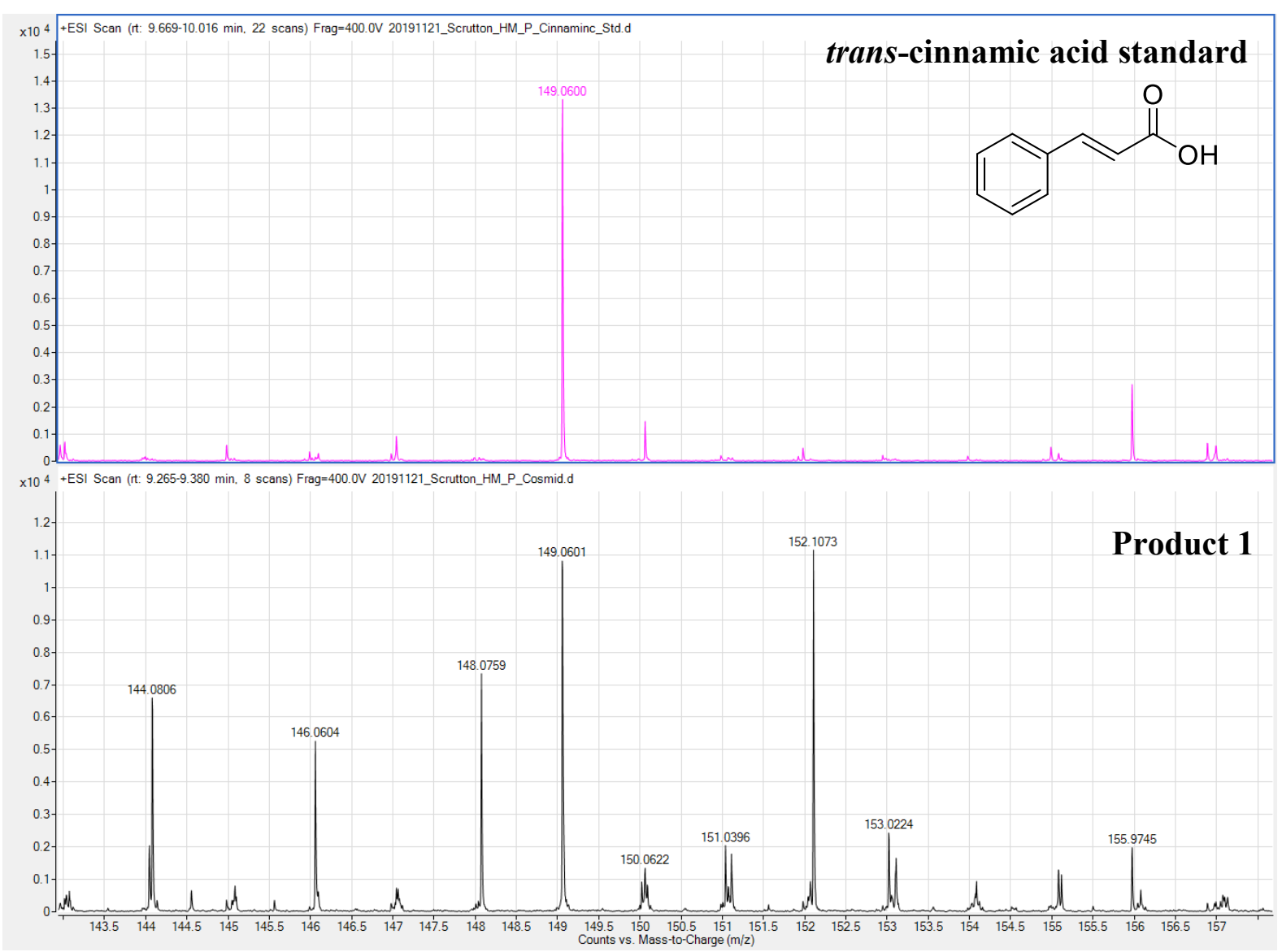


2,4,6-octatrienoic acid MS data (product 2 in Fig 3A)

(negative ion mode)

The calculated monoisotopic mass of 2,4,6-octatrienoic acid is $138.06807\left(\mathrm{C}_{8} \mathrm{H}_{10} \mathrm{O}_{2}\right)$

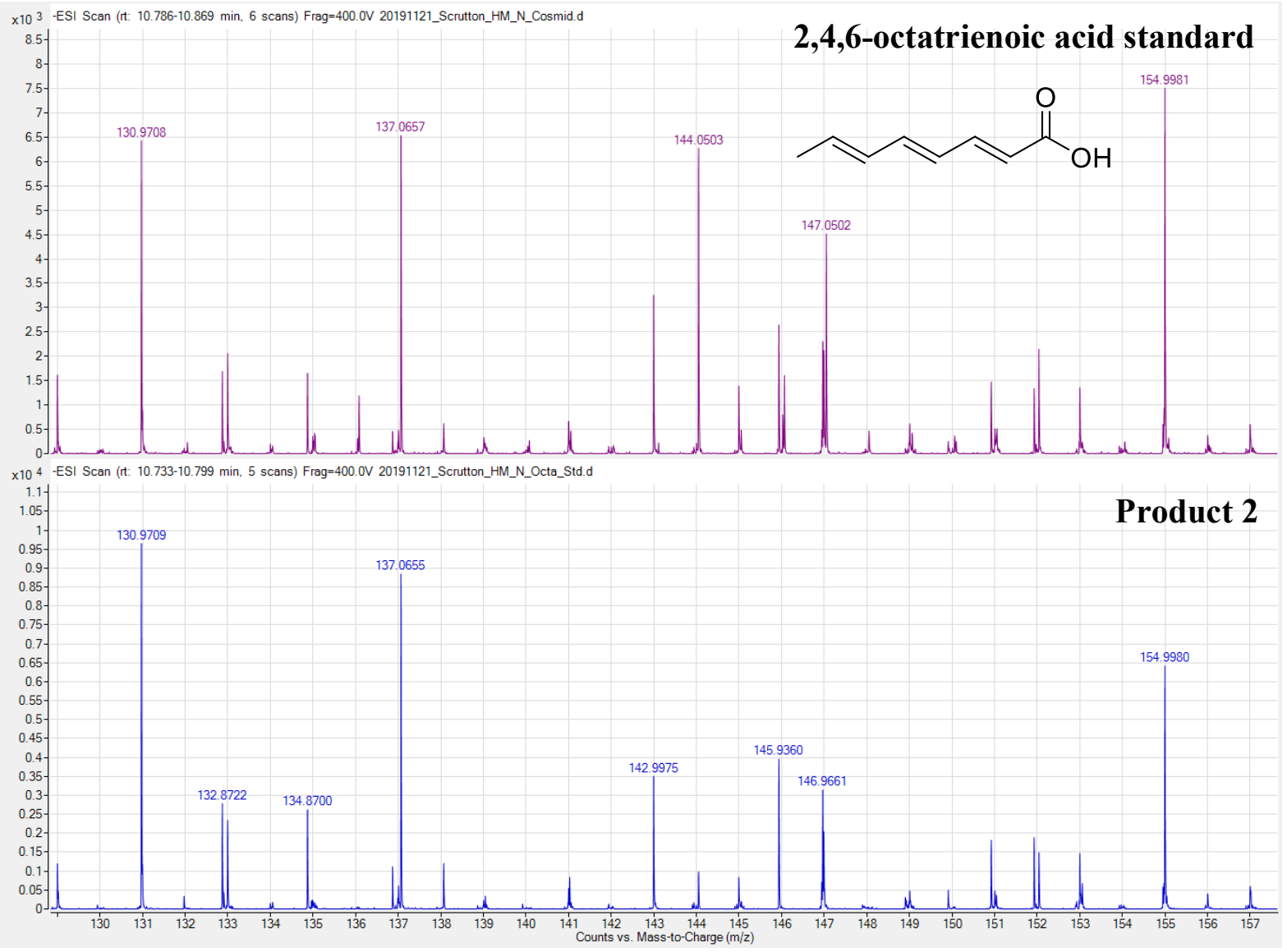


2,4,6-octatrienoic acid MS data (product 2 in Fig 3A)

(positive ion mode)

The calculated monoisotopic mass of 2,4,6-octatrienoic acid is $138.06807\left(\mathrm{C}_{8} \mathrm{H}_{10} \mathrm{O}_{2}\right)$

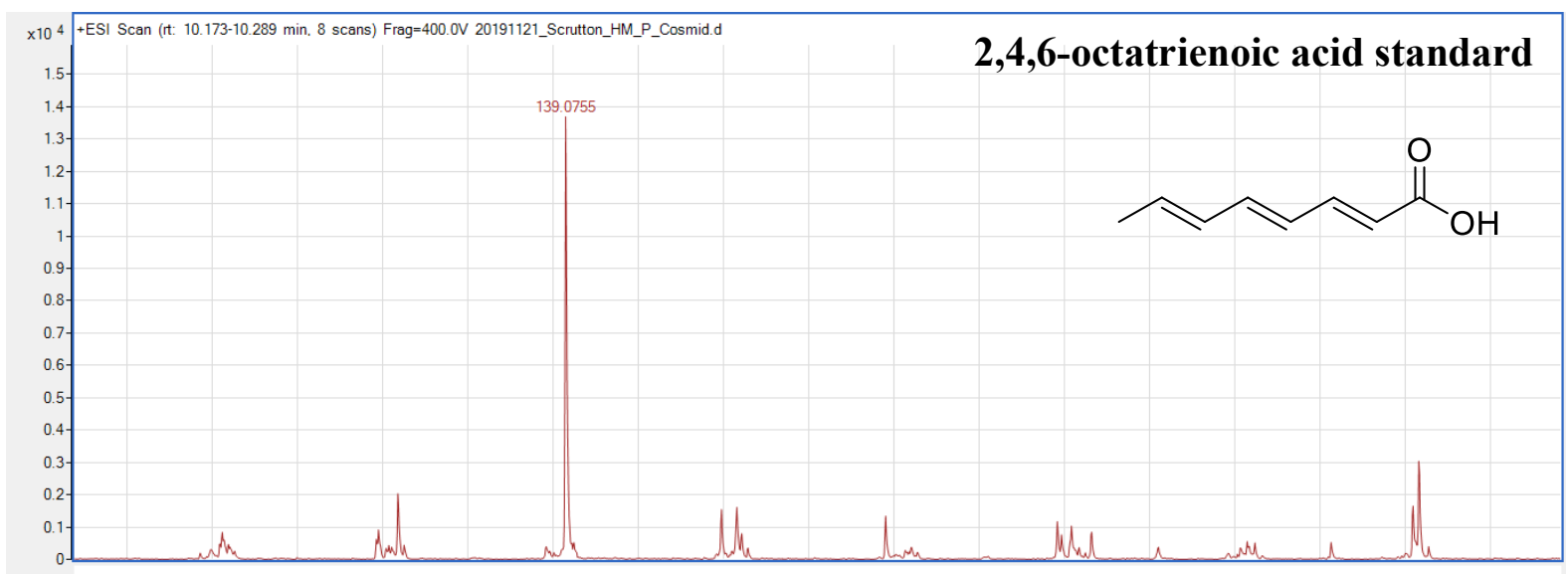

x10 4 +ESI Scan (it: 10.496-10.529 min, 3 scans) Frag=400.0V 20191121_Scrutton_HM_P_Octa_Std.d

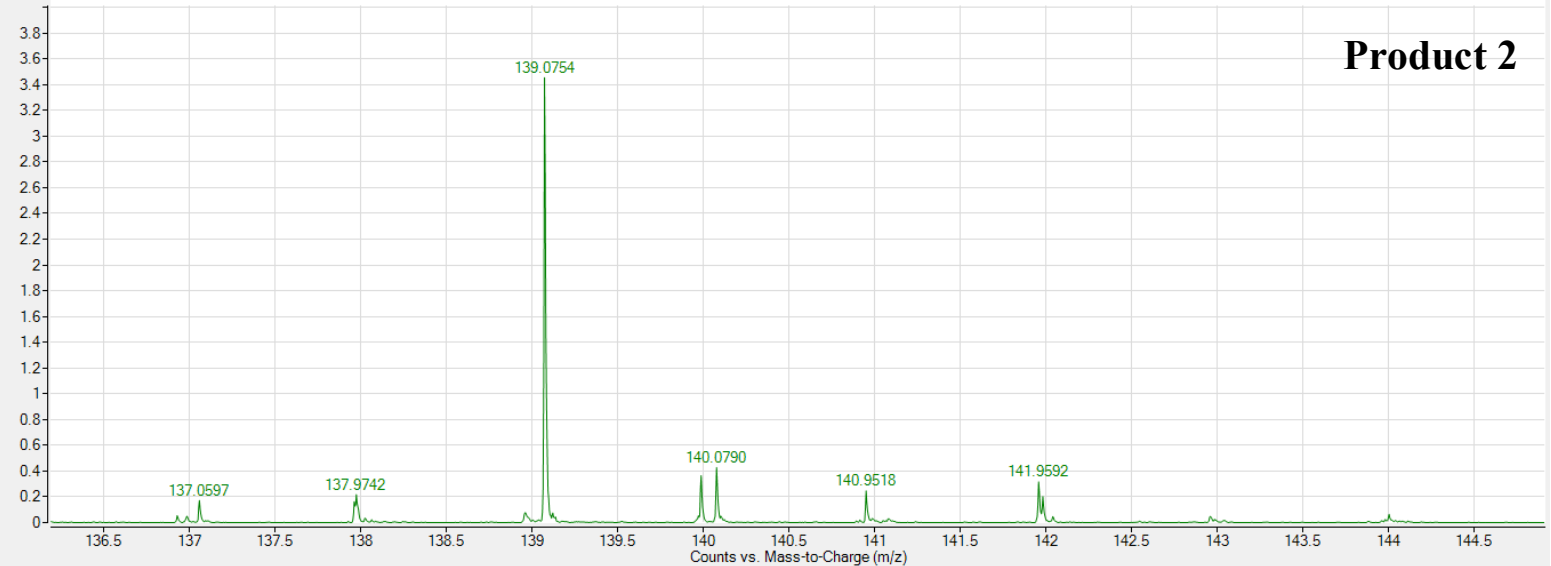




\section{Andrimid MS data (product 4 in Fig 3A)}

(positive ion mode)<smiles>C/C=C/C=C/C=C/C(=O)N[C@@H](CC(=O)NC1C(=O)[C@@H]2C(=O)NC(=O)[C@H]2CC1C)c1ccccc1</smiles>

andrimid

The calculated monoisotopic mass of andrimid is $479.24200\left(\mathrm{C}_{27} \mathrm{H}_{33} \mathrm{~N}_{3} \mathrm{O}_{5}\right)$

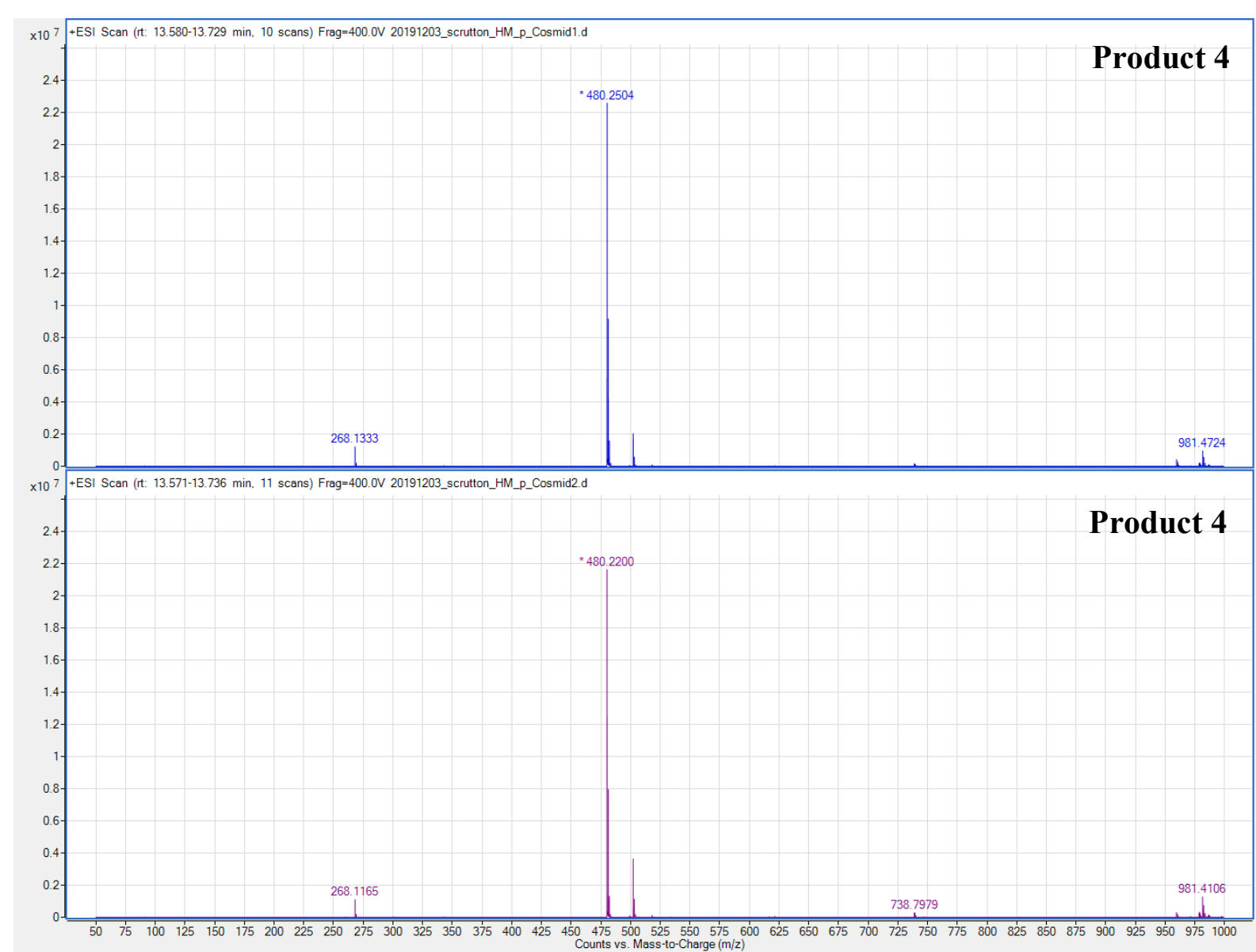

The data shows replicates produced from two cultures (two colonies) 


\section{MS data (product 3 in Fig 3A)}

(positive ion mode)

\section{(unidentified)}

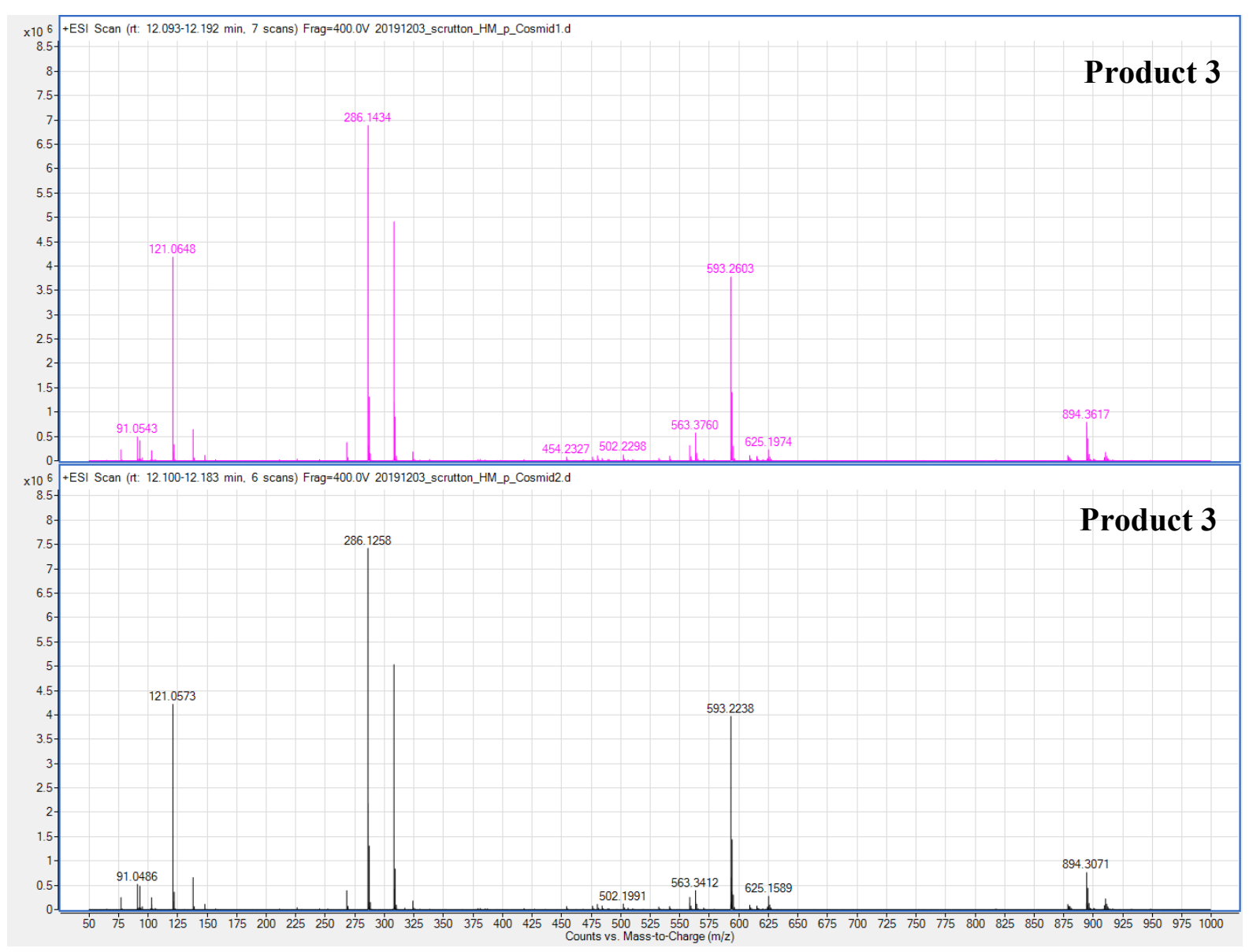

The data shows replicates produced from two cultures (two colonies) 


\subsection{GC/MS data}

Figure S9. Mass spectra of hepta-1,3,5-triene product (in Fig 3B, Fig 3 C) analysed by GC/MS in the gaseous phase.

hepta-1,3,5-triene product in Fig $3 B$ produced from the del_AdmH-AdmP construct expression in XL1-Blue MR
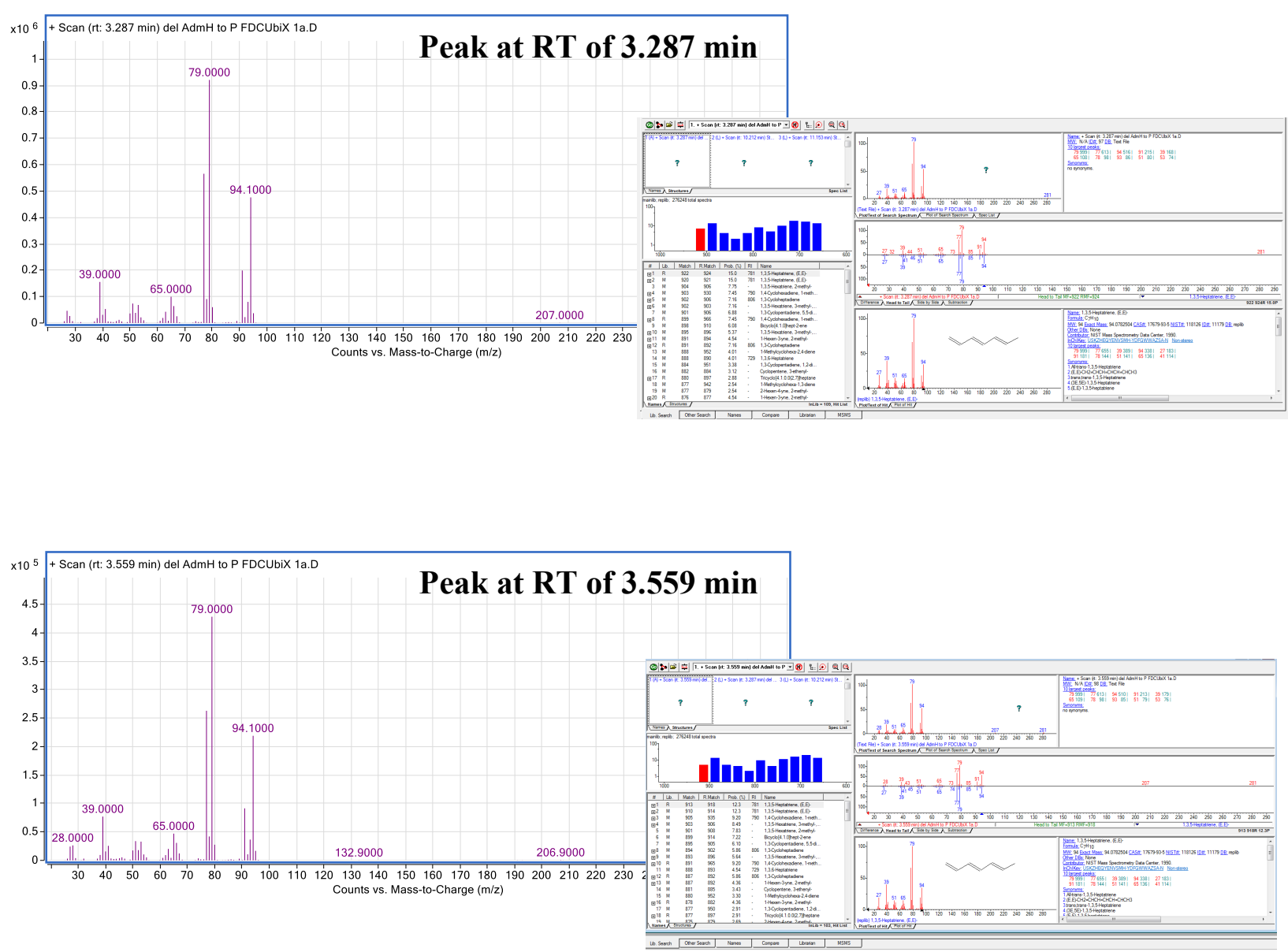
hepta-1,3,5-triene product in Fig 3C produced from decarboxylation of standard 2,4,6octatrienoic acid by Fdc1
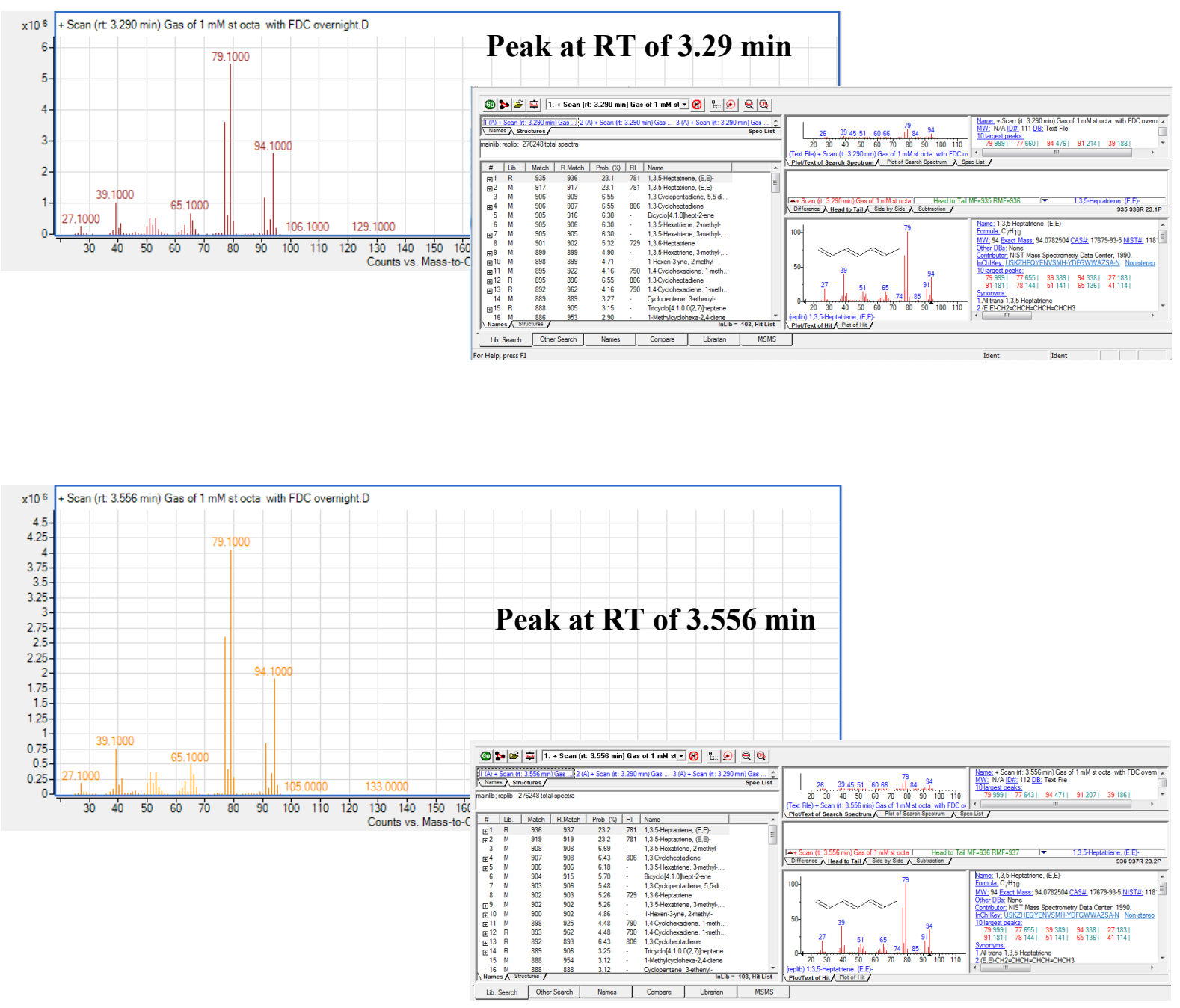


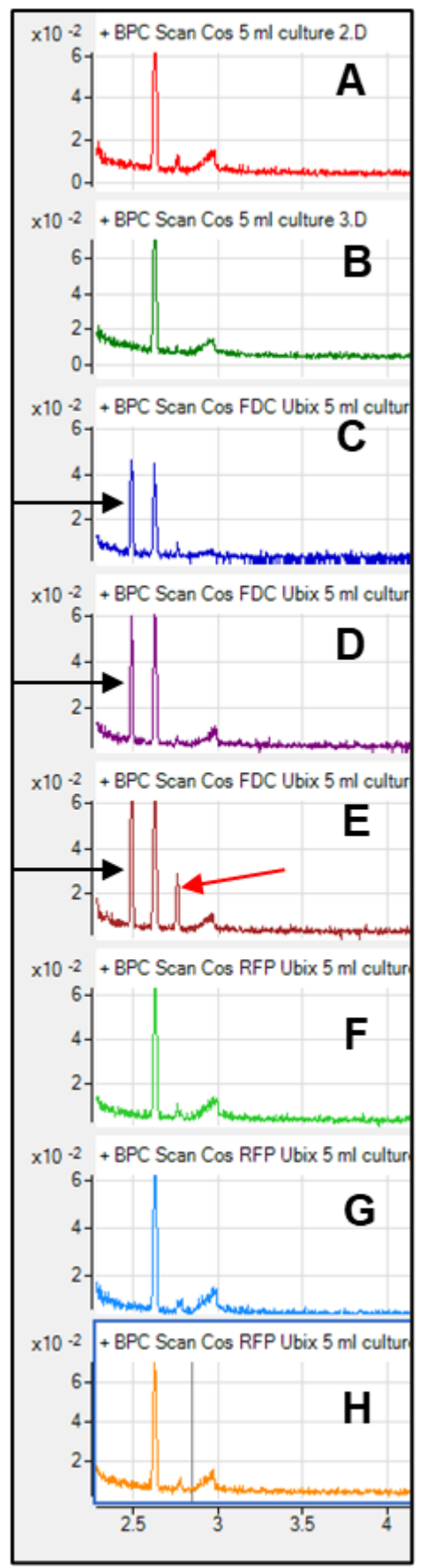

hepta-1,3,5-triene, RT of $2.501 \mathrm{~min}$
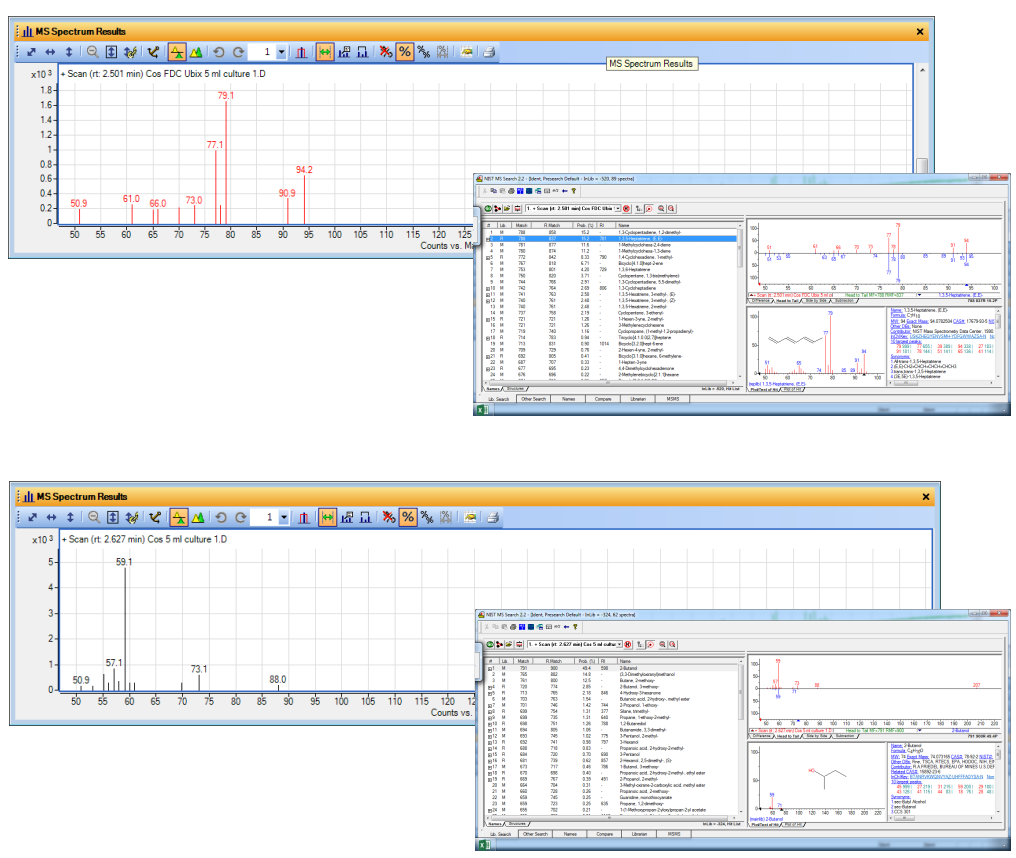

cycloheptatriene, RT of $2.766 \mathrm{~min}$

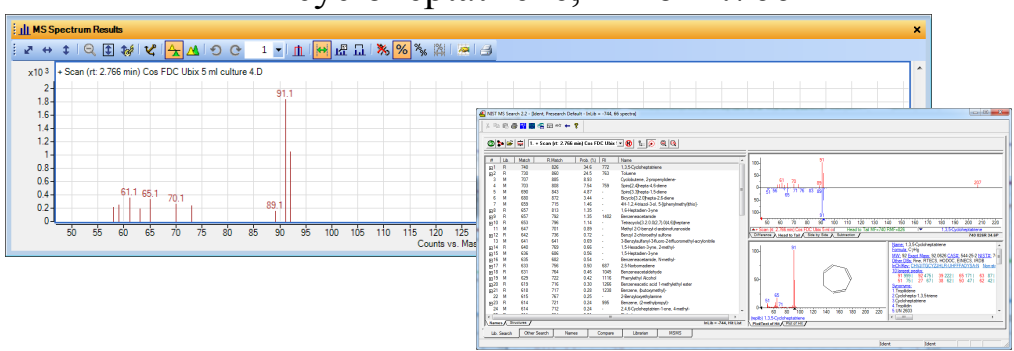

Figure S10. Mass spectra of hepta-1,3,5-triene product analysed by GC/MS in the liquid phase. Replicates of $5 \mathrm{ml}$ culture of the cosmid (A-C), cosmid with Fdc1/UbiX (D-F) and control UbiX in $\mathrm{pBbA1C}$-RFP vector $(\mathrm{G}-\mathrm{H})$ were grown in sealed $20 \mathrm{ml}$ vials at $30^{\circ} \mathrm{C}$ overnight $(16$ hours after IPTG induction in case of cosmid with Fdc1/UbiX.) All cultures were grown in parallel. 5 $\mathrm{ml}$ cultures were extracted with $2 \mathrm{ml}$ ethyl acetate, dried with magnesium sulphate and injected in the GC/MS ( $1 \mu 1$ injection volumes). GC/MS conditions as in the "Methods" section. Black arrows: hepta-1,3,5-triene, RT of $2.501 \mathrm{~min}$ (in C-E), red arrow: cycloheptatriene, RT of 2.766 $\min ($ in $\mathrm{E})$. 

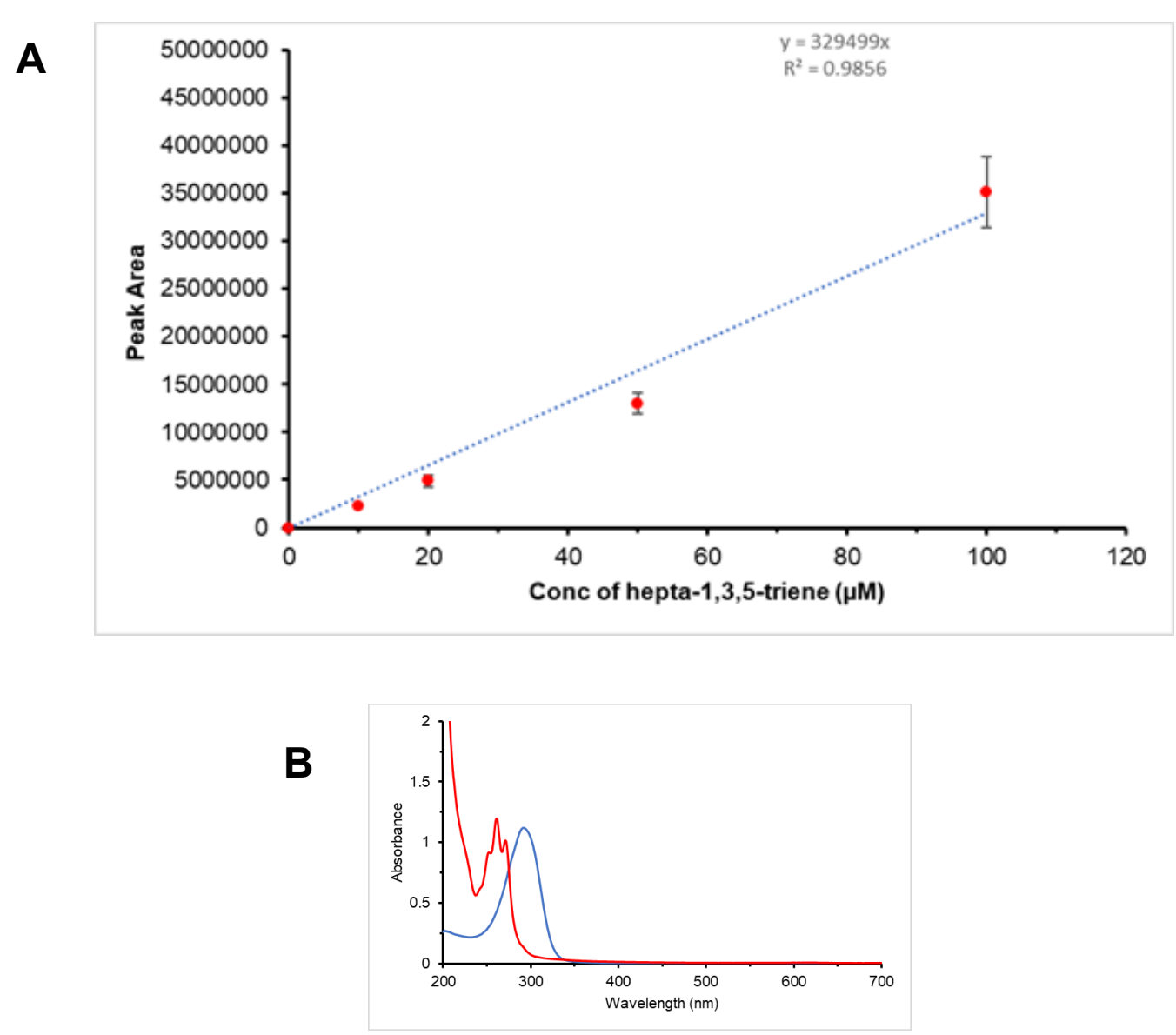

Figure S11. (A): Standard curve of hepta-1,3,5-triene measured by GC/MS. Conditions: four concentrations of hepta-1,3,5-triene $(10-100 \mu \mathrm{M})$ were prepared by decarboxylating known concentrations of 2,4,6-octatrienoic acid by purified Fdc1 in situ in $50 \mathrm{mM}$ sodium phosphate buffer at $\mathrm{pH} 6.5$ at $30^{\circ} \mathrm{C}$. The decarboxylation reactions were carried out in the reservoirs of tight Hamilton syringes ( $2 \mathrm{ml}$ reaction each) without introducing gas phase to ensure the produced hepta-1,3,5-triene is contained in the liquid phase and the reaction was also followed in parallel by UV-Vis spectrophotometric measurements to follow the complete conversion of acid to alkene (disappearance of the acid peak at $300 \mathrm{~nm}$ ). The produced hepta-1,3,5-triene was spiked ( $1 \mathrm{ml}$ each to be spiked into $4 \mathrm{ml} \mathrm{LB})$ in $20 \mathrm{ml}$ sealed GC vials and measured by GC/MS measurements to mimic the conditions used in measuring the biological replicates. Duplicate measurements were obtained for each concentration (10-100 $\mu \mathrm{M}$ concentrations). The various concentrations were measured and the combined data were used to plot the standard curve (as in A). GC/MS conditions as described in the "Methods" section (injection volumes: $1 \mathrm{ml}$ by autosampler injection). (B): Spectrophotometric analysis to follow the full conversion of 2,4,6octatrienoic acid to hepta-1,3,5-triene on preparing hepta-1,3,5-triene in situ. Conditions: 2,4,6octatrienoic acid $(0.5 \mathrm{mM})$ and $\mathrm{Fdcl}(0.4 \mu \mathrm{M})$ in $50 \mathrm{mM}$ sodium phosphate buffer $\mathrm{pH} 6.5$ at $30^{\circ} \mathrm{C}$. $0.5 \mathrm{mM}$ concentration was the highest concentration used (when spiked into a total volume of $5 \mathrm{ml}$ to give $100 \mu \mathrm{M}$ ). The decarboxylation reaction was diluted 1:20 with buffer to be measured before (blue) and after the completion of the reaction (red). Full conversion of 2,4,6-octatrienoic acid to hepta-1,3,5-triene was achieved in 2 minutes under the conditions used. 


\subsection{HPLC Analysis}

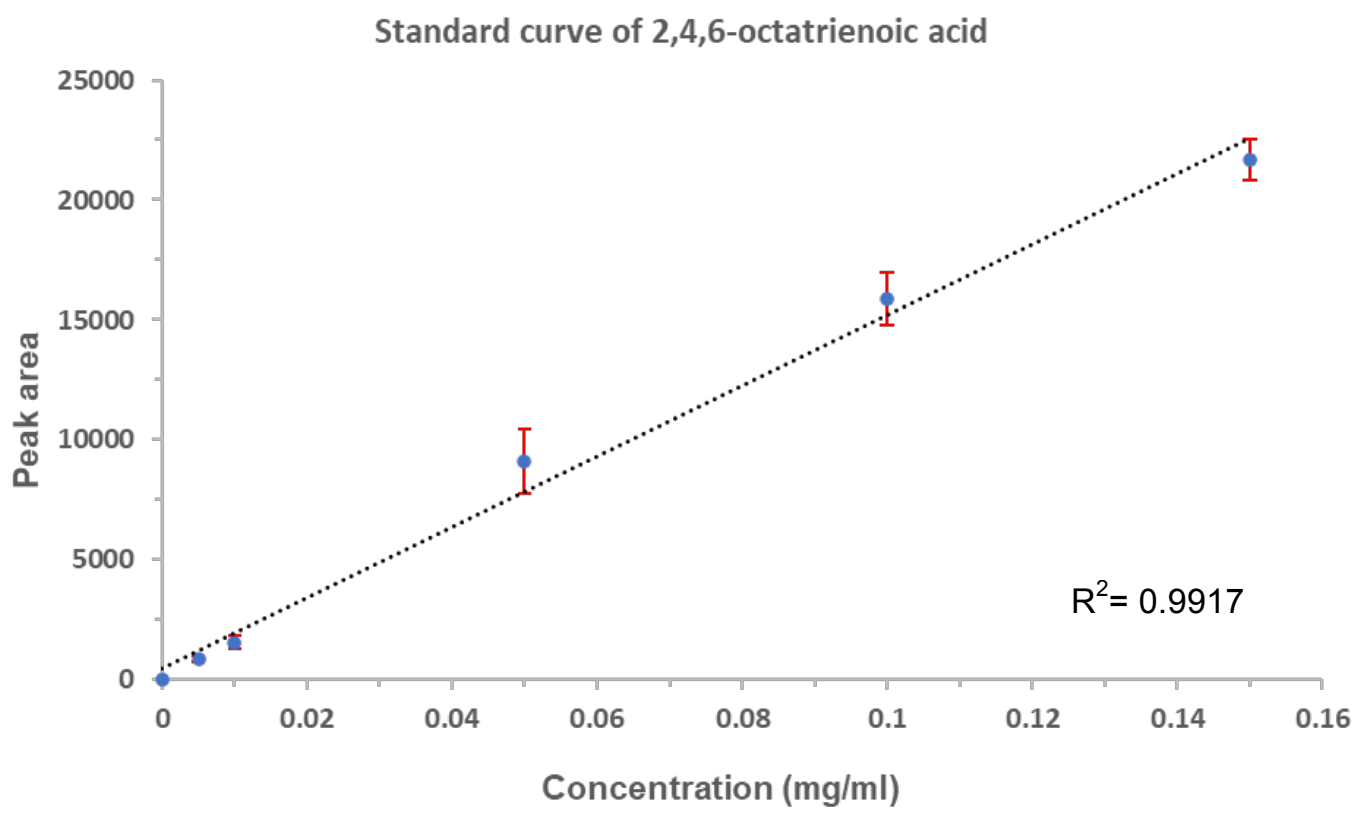

Figure S12. Standard curve of 2,4,6-octatrienoic acid measured by HPLC. Conditions: two sets of 5 concentrations of 2,4,6-octatrienoic acid $(0.005,0.01,0.05,0.1,0.15 \mathrm{mg} / \mathrm{ml}$ in $50 \%$ methanol $/ \mathrm{H}_{2} \mathrm{O}$ HPLC grade) were measured each in triplicates. The combined data were used to plot the standard curve. HPLC conditions as described in the "Methods" section (injection volumes: $50 \mu 1$ ). 

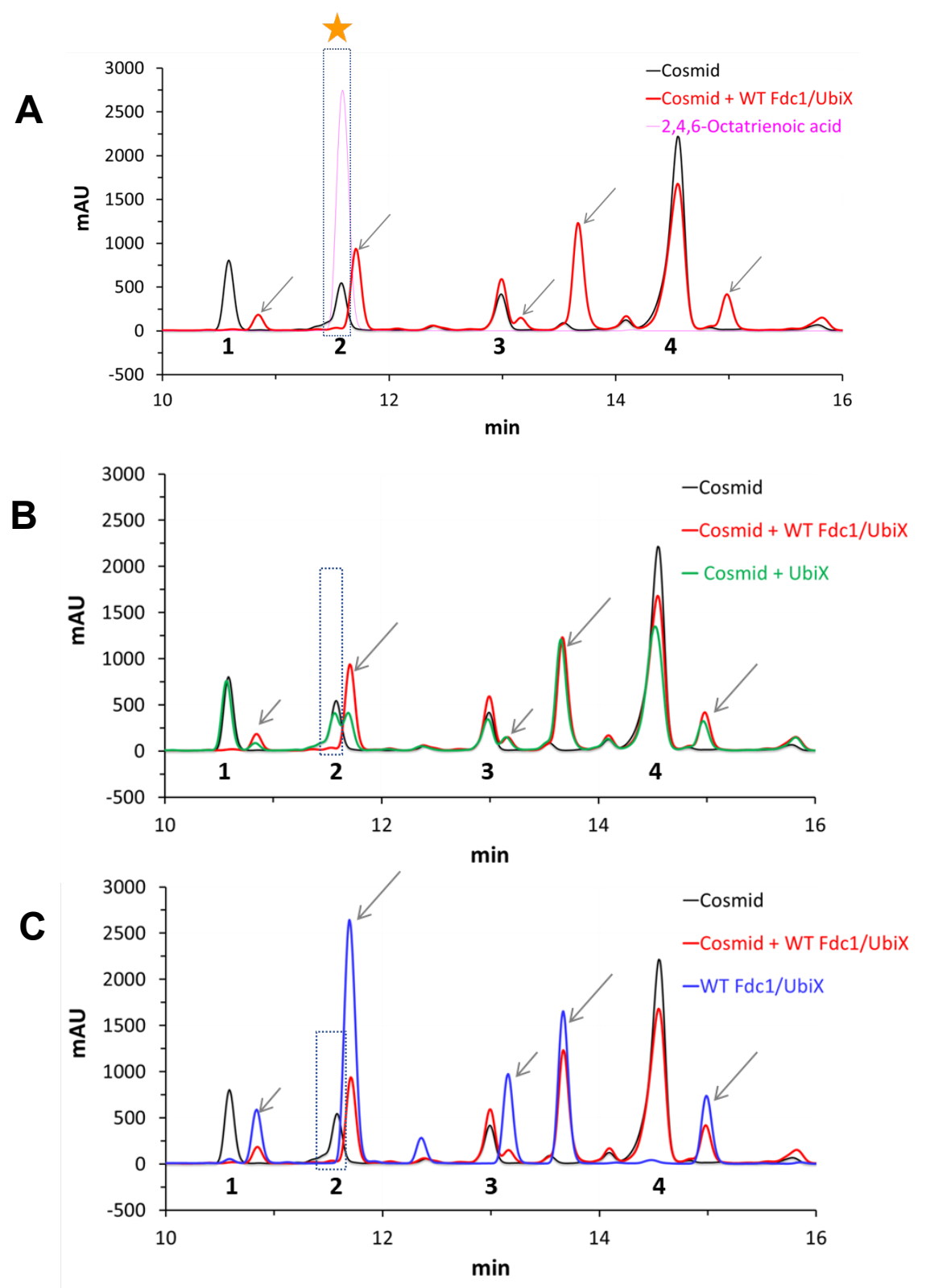

Figure S13. HPLC analysis showing the Fdc1 catalysed decarboxylation of the 2,4,6octatrieonic acid product of cosmid $2194 \mathrm{C} 1$ in vivo. HPLC analysis of the products obtained from the expression of cosmid 2194C, the co-expression of cosmid $2194 \mathrm{C} 1$ and Fdc1/UbiX, the co-expression of cosmid $2194 \mathrm{C} 1$ and UbiX and the expression of Fdc1/UbiX in XL1-Blue MR cells as described in the "Methods" section. The chromatograms were overlapped as in panels $\mathrm{A}, \mathrm{B}$ and $\mathrm{C}$ to illustrate the disappearance of 2,4,6-octatrienoic acid (product 2, also in a dotted box) by Fdc1/UbiX. The peak of 2,4,6-octatrienoic acid standard was shown as a guide to clarify the results. The arrows highlighted the various peaks arising from Fdc1/UbiX or UbiX constructs. Due to the complexity of the data/peaks, this figure displays the peaks between RT of 10 -16 minutes that shows the four major products of the cosmid for simplification. Conditions: as explained in the "Methods" section. 


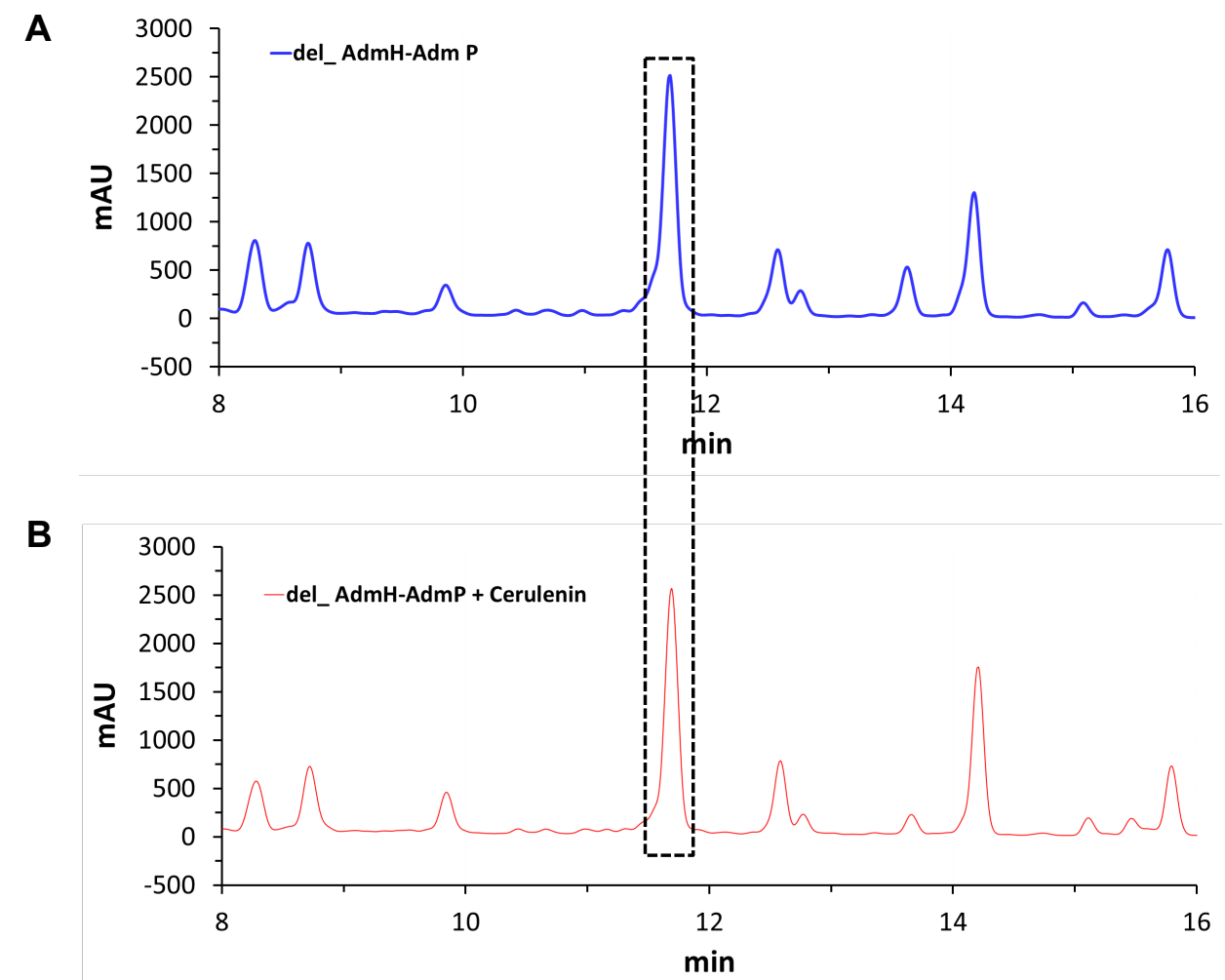

Figure S14. HPLC analysis of the products from the expression of the del_AdmH-AdmP construct in XL1-Blue MR in the absence (A) and presence of cerulenin (20 $\mu \mathrm{g} / \overline{\mathrm{ml}}$ culture) (B). Conditions: cerulenin was added to the cell culture at an $\mathrm{OD}_{600 \mathrm{~nm}}$ of 0.6 and cultures were grown overnight at $30^{\circ} \mathrm{C}$. HPLC analysis as described in the "Methods" section. 2,4,6-octatrienoic acid titer is $12.8 \mathrm{mg} / \mathrm{L}$ in presence of cerulenin compared to $13.2 \mathrm{mg} / \mathrm{L}$ in its absence. The biomass was less in presence of cerulenin: $\mathrm{OD}_{600 \mathrm{~nm}}$ of 4.36 compared to 6.66 in absence of cerulenin. 


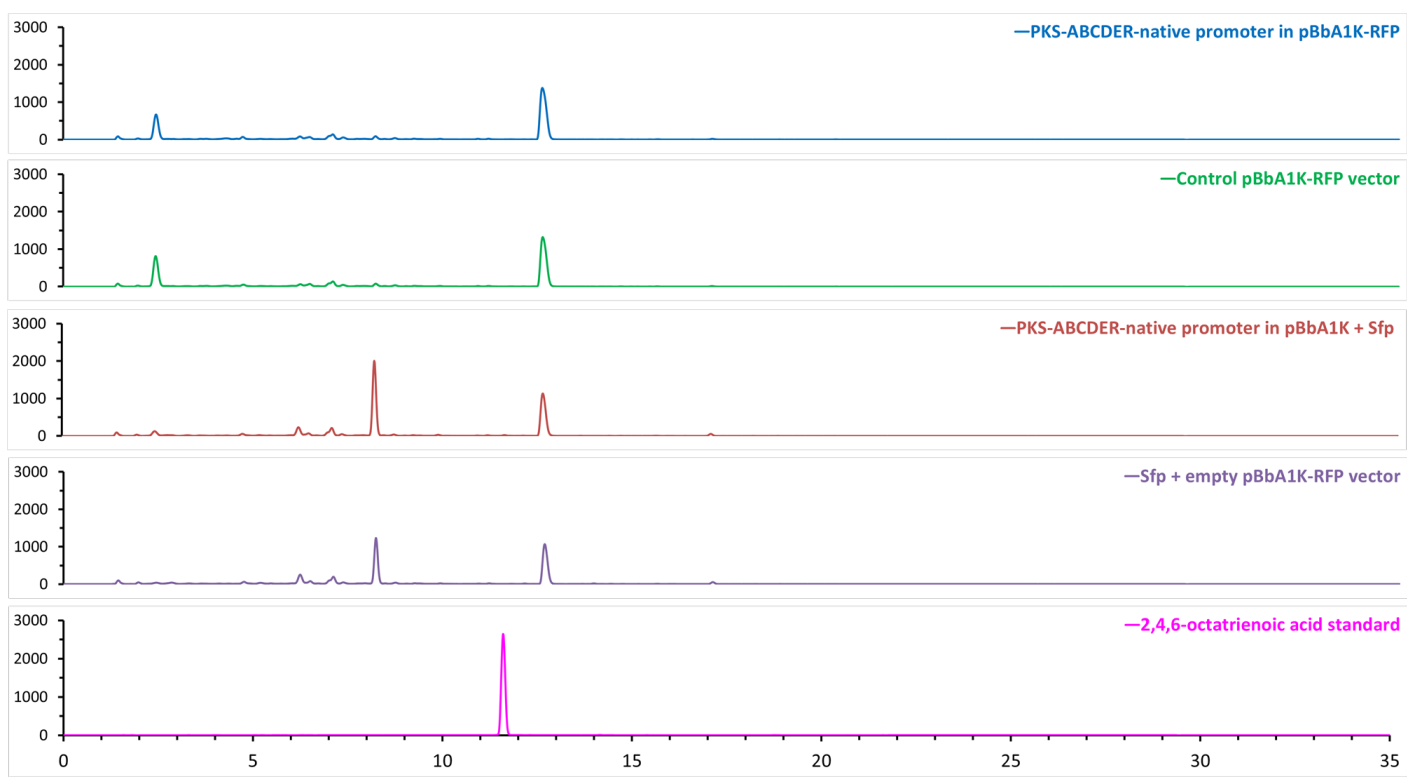

Figure S15. HPLC analysis of the products from the expression of the PKS-ABCDER-native promoter construct in pBbA1K-RFP vector and from the co-expression with Bacillus subtilis Sfp in BL21(DE3) cells. The products of the control vectors were also analysed and displayed for comparison with also 2,4,6-octatrienoic acid standard. Conditions as described in section 1.4. HPLC conditions as described in the "Methods" section. 


\section{References}

(1) Hertweck, C., Luzhetskyy, A., Rebets, Y., and Bechthold, A. (2007) Type II polyketide synthases: gaining a deeper insight into enzymatic teamwork. Nat. Prod. Rep. 24, 162-190. https://doi.org/10.1039/B507395M

(2) Zhang, W., and Tang, Y. (2009) In vitro analysis of type II polyketide synthase, In Methods in Enzymology, pp 367-393. Academic Press. https://doi.org/10.1016/S0076-6879(09)04616-3

(3) Lee, T. S., Krupa, R. A., Zhang, F., Hajimorad, M., Holtz, W. J., Prasad, N., Lee, S. K., and Keasling, J. D. (2011) BglBrick vectors and datasheets: A synthetic biology platform for gene expression. J. Biol. Eng. 5, 12. https://doi.org/10.1186/1754-1611-5-12

(4) Gahloth, D., Dunstan, M. S., Quaglia, D., Klumbys, E., Lockhart-Cairns, M. P., Hill, A. M., Derrington, S. R., Scrutton, N. S., Turner, N. J., and Leys, D. (2017) Structures of carboxylic acid reductase reveal domain dynamics underlying catalysis. Nat.Chem. Biol.13, 975-981. https://doi.org/10.1038/nchembio.2434

(5) Jin, M., Fischbach, M. A., and Clardy, J. (2006) A biosynthetic gene cluster for the acetylCoA carboxylase inhibitor andrimid. J. Am. Chem. Soc. 128, 10660-10661. https://doi.org/10.1021/ja063194c 